\title{
Comparison of planar DMSA scan with an evaluation based on SPECT imaging in the split renal function assessment
}

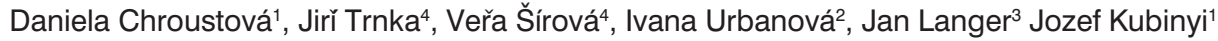 \\ ${ }^{1}$ Department of Nuclear Medicine, General University Hospital and ${ }^{1 s t}$ Faculty of Medicine, Charles University Prague, Czech Republic \\ 2Department of Pediatrics, University Hospital Bulovka, Prague, Czech Republic \\ ${ }^{3}$ Clinic of Pediatrics and Adolescent Medicine, General University Hospital and ${ }^{1 s t}$ Faculty of Medicine, Charles University Prague, \\ Czech Republic \\ ${ }^{4}$ Department of Radiation Protection, General University Hospital Prague, Czech Republic
}

[Received 20 IV 2015; Accepted 10 XI 2015]

\begin{abstract}
BACKGROUND: Validation of the ${ }^{99 m}$ TC-DMSA planar scintigraphy accuracy for split renal function assessment and comparison with evaluation based on SPECT imaging both with and without CT attenuation correction.

MATERIALS AND METHODS: For split renal function assessment two methods were used: A) planar scintigraphy based on anterior and posterior projections using correction for kidney depth calculated by the geometric mean; B) semi-quantitative evaluation based on SPECT (B1) and attenuation-corrected SPECT/CT (B2) images using locally developed software for kidney segmentation and voxel-based analysis. All three methods were performed with a phantom simulating body including pair of kidneys. For patient study methods A and B1 were applied on a group of 140 children and adolescents with various renal diseases. Renal function ratios were compared both mutually and with physically measured activity ratios in the phantom.

RESULTS: Method A provided results which were closest to measured reference values (average absolute difference of 0.9 percentage points [pp]). Method B1 was noticeably worse (2.1pp), whereas attenuation correction (B2) improved tomography results considerably (1.3 pp). The superiority of planar imaging could be caused among others by differences in creation of planar range of interest compared to tomographic volume of interest. However all the differences were under the threshold of any clinical importance. The comparison between method A and B1 based on patient study also showed differences mostly of none clinical importance.

CONCLUSION: Routine evaluation of split renal function using planar technique with correction of the kidney depth is at least equivalent to tomographic evaluation, and there is no need to update the established clinical practice.
\end{abstract}

KEY words: static renal ${ }^{99 m}$ TC-DMSA scintigraphy, 3D SPECT method analysis, split renal function ratio, childhood

Nuclear Med Rev 2016; 19, 1: 12-17

\section{Background}

Renal cortical scintigraphy using Dimercaptosuccinic Acid labeled by Technetium-99m (99mTc-DMSA) is widely used in pediatric nephro-urology practice [1-12]. Under usual conditions the renal parenchyma is visualized without interference from the pelvicalyceal system and good images and a good estimation of the function can be obtained [1]

Such investigation is performed not only for good imaging of the renal parenchyma but also for quantification of the split renal function, which has major clinical importance. Determination of

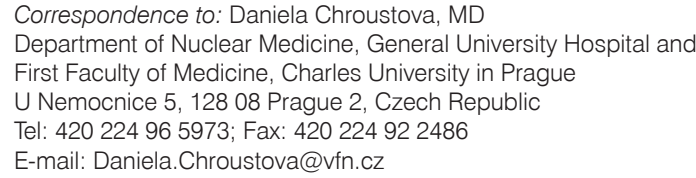

the left to right ${ }^{99 m} \mathrm{Tc}$-DMSA uptake ratio is considered as the basic and valuable parameter and routinely is calculated from planar scintigrams.

The relative ${ }^{99 m}$ Tc-DMSA renal uptake may be used as an index of the renal function and to evaluate the change in renal function at follow up. Quantification can be performed using a couple of methods: 1) evaluation based on the posterior view only, with or without compensation for kidney depth; 2) evaluation based on both anterior and posterior views using their geometric mean to compensate for kidney depth. The latter method is used more often and it is also usually considered as more valid.

Current expansion of tomographic imaging caused many nuclear medicine imaging methods to convert from planar into tomography. Is it also meaningful for split renal function assessment? Does tomographic study introduce any clinical benefit over planar imaging in this case? There was a primary assumption that the most accurate values of the uptake ratio are obtained from 
Single Photon Emission Computed Tomography (SPECT) examination. The assumption was based on the fact that SPECT imaging generally provides more accurate description of spatial distribution of activity as well as better contrast of organs of our interest. A phantom measurement was used to verify this assumption. Except the phantom measurement a patient study was performed to assess the differences between planar and SPECT imaging in clinical practice.

\section{Materials and methods}

\section{Phantom measurements}

There had been constructed a phantom model, illustrated on Figure 1, consisting of NEMA/IEC (NU 2-2007) body phantom (height $x$ width $x$ depth $=24.1 \times 30.5 \times 24.1 \mathrm{~cm}$ ) and a pair of plastic bottles, which were of size and volume similar to human kidney (height $10 \mathrm{~cm}$, diameter $4 \mathrm{~cm}$ ). The pair of bottles was located within the phantom in two geometries - firstly simulating normal localization (Figure 1 - top), secondly simulating atypical localization of renal dystopia (Figure 1 - bottom). In the normal localization the bottles were put to the same height level regarding the cranio-caudal direction as well as in the same depth regarding antero-posterior view. Dystopic (pathologic) geometry was simulated by the same bottles positioned differently, so the left bottle was placed $5 \mathrm{~cm}$ below and $8 \mathrm{~cm}$ ventrally to the right one. Bottles were repeatedly filled with different activities of ${ }^{99 \mathrm{~m}} \mathrm{Tc}$, according to Table 1 simulating different renal uptake ratios. The phantom background compartment was filled by $20 \mathrm{MBq}$ of ${ }^{99 \mathrm{~m}} \mathrm{Tc}$.

Two different acquisitions were performed: a) simultaneous planar anterior and posterior projections, stop condition $300 \mathrm{kcts}$, low energy high resolution (LEHR) collimator, matrix $256 \times 256$ pixels, zoom 1.6, and b) SPECT acquisition consisting of 120 projections, 25 s per projection, LEHR collimator, matrix $128 \times 128$ pixels, zoom 1.6. Data were reconstructed using Ordered Subsets Expectation Maximization (OSEM) iterative algorithm (2 iterations, 10 subsets, Hanning post-filter). The reconstruction was performed both without attenuation correction - method B1 and with Computed Tomography Attenuation Correction (CTAC) - method B2.

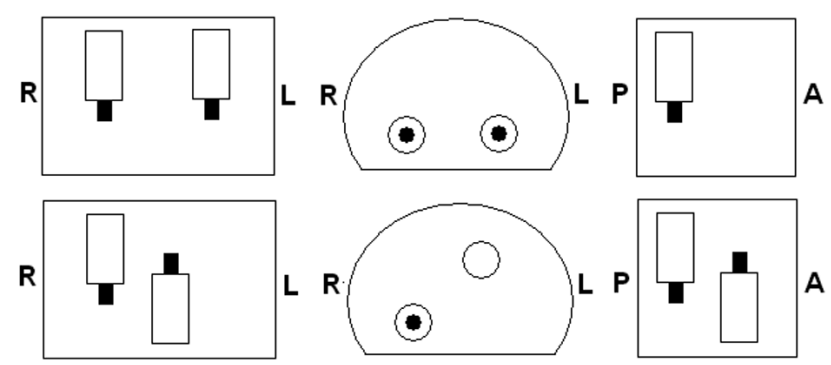

Figure 1. Phantom construction. NEMA/IEC (NU 2-2007) body phantom was filled with water, standard inserts were removed, and two plastic bottles were added. Top line demonstrates the normal kidney localization, the bottom line simulates kidney dystopia. The bottles were in default placed $3 \mathrm{~cm}$ from the posterior wall of the phantom. For the simulation of dystopia the left bottle was shifted by $8 \mathrm{~cm}$ ventrally and by $5 \mathrm{~cm}$ lower. The images represent coronal (left), transversal (middle), and sagittal (right) slices respectively
Table 1. Various activities in the bottles simulating different ratios of renal uptake by different geometries. Normal geometry was represented by two bottles in the same height level and in the same distance from the rear wall of the phantom. Dystopic (pathologic) geometry was simulated by the same bottles positioned differently so the left bottle was placed $5 \mathrm{~cm}$ below and $8 \mathrm{~cm}$ ventrally to the right one

\begin{tabular}{cccccc}
\multicolumn{2}{c}{$\begin{array}{c}\text { Activity in the bottle } \\
\text { [MBq] }\end{array}$} & & \multicolumn{2}{c}{$\begin{array}{c}\text { Relative activity* } \\
\text { 100\% }\end{array}$} & Geometry \\
\cline { 1 - 2 } \cline { 5 - 6 } Right & Left & & Right & Left & \\
\cline { 5 - 6 } 49.3 & 49.3 & & 50 & 50 & Normal \\
49.3 & 50.6 & & 49 & 51 & Dystopic \\
60.0 & 39.9 & & 60 & 40 & Normal \\
60.0 & 39.8 & & 60 & 40 & Dystopic \\
68.9 & 31.1 & & 69 & 31 & Normal \\
68.9 & 31.0 & & 69 & 31 & Dystopic \\
79.1 & 20.1 & & 80 & 20 & Normal \\
79.1 & 19.5 & 80 & 20 & Dystopic \\
88.1 & 10.1 & 90 & 10 & Normal \\
88.1 & 10.0 & 90 & 10 & Dystopic \\
\hline
\end{tabular}

\section{Patient study}

A total of 140 patients (53 boys and 87 girls aged from 5 to 18 years) with various renal diseases and congenital abnormalities were examined. Fifty-seven of them had a pathological split renal function (more than 55/45\%) due to reduced renal function, different size or abnormal renal position. The administered activity (40-100 MBq) was calculated according to European Association of Nuclear Medicine (EANM) dosage card for children [13]. Planar renal scintigraphy was performed 2 hours after ${ }^{99 \mathrm{~m} T c-D M S A}$ i.v. administration and followed by SPECT acquisition. The patient acquisition protocols for both planar and SPECT imaging were the same as in the case of phantom experiment. In case of youngest children with reduced administered activity, the acquisition time for SPECT was prolonged to 30s per projection. Moreover, no CT was performed due to young patients so no attenuation correction was available. Therefore, only method A and B1 regarded the patient study.

All measurements and acquisitions were performed on GE Infinia Hawkeye gamma camera.

\section{Calculation}

Split renal function in both phantom and patient study was determined by an experienced physician using the following methods according to the type of acquisition:

\section{Planar scintigraphy (method A)}

There were manually created regions of interest $(\mathrm{ROI})$ on both anterior and posterior projections covering each kidney and its corresponding background (Figure 2). Firstly, all kidney ROls were corrected for background by subtracting average value of corresponding background ROI. The total kidney counts were determined using the geometric mean of anterior and posterior projections to compensate for kidney depth. The following formulas were applied for the split renal function calculation: 
ANT

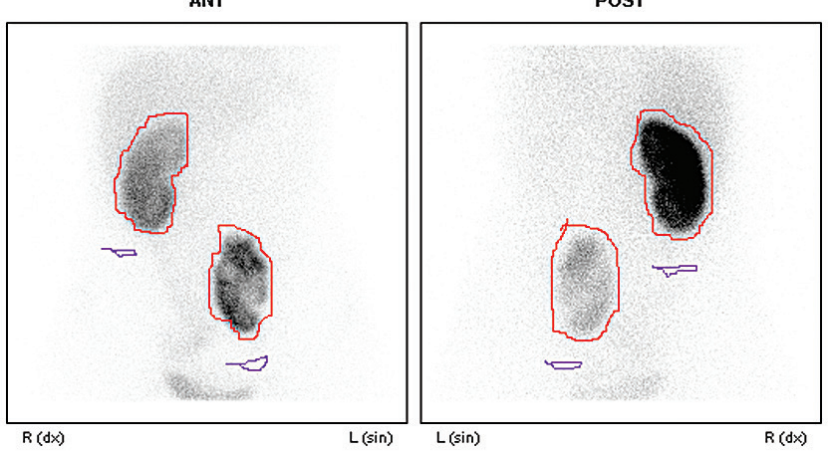

Figure 2. Example of split renal function assessment from planar images. The red ROls cover kidneys, the purple ROls represent background in 15 years old girl with dystopic malformed left kidney. The geometric mean calculation was employed to compensate for kidney depth. The separate renal ratio in this example was $25 / 75 \%$ on behalf of the right kidney

(Equation 1) $L=\frac{\sqrt{L_{p_{\text {ost }}} \cdot L_{\text {Ant }}}}{\sqrt{L_{\text {post }} \cdot L_{\text {ant }}}+\sqrt{R_{\text {post }} \cdot R_{\text {Ant }}}} \cdot 100 \%$

(Equation 2) $\quad R=\frac{\sqrt{R_{p_{o s t}} \cdot R_{\text {Ant }}}}{\sqrt{L_{\text {post }} \cdot L_{\text {ant }}}+\sqrt{R_{p_{\text {ost }}} \cdot R_{\text {Ant }}}} \cdot 100 \%$

where $L_{\text {Ant }}, L_{\text {Post }}, R_{\text {Ant }}$, and $R_{\text {Post }}$ were counts in corresponding ROIs previously corrected for background.

\section{SPECT (method B1) and SPECT CTAC (method B2)}

For tomography images assessment locally developed software was used. It employs the following algorithm for proper kidney segmentation and counts extraction from desired volumes of interest (VOIs).

A VOI consists of several selected voxels. Voxels belonging to the $\mathrm{VOI}$ are determined by a spatial binary mask — a three-dimensional lattice of true/false values where each value corresponds to particular voxel in image data. So the VOI consists of voxels with true mask value. The binary mask may be determined either by an intensity threshold or by some geometric parameters. In case of the intensity threshold a user enters an arbitrary number so only the voxels with higher intensity than that number get true mask value and contribute to the VOI (and vice versa). This step is sufficient to separate kidneys from the background but for separation both kidneys from each other it is necessary to apply some spatial confinement. Generally, it is possible to construct a geometrical shape and let all voxels inside the shape have true mask value (and vice versa). In our case the user selects a particular voxel (preferably close to the middle of the kidney) which represents the center of a sphere and then the user enters the radius of the sphere. All voxels inside the sphere have true mask value, whereas those outside the sphere are all false. Each kidney has its own spherical mask and so it is spatially confined (encapsulated within the sphere). As the final step the software intersects (takes the common part of) each spherical mask with the intensity mask. The true-value content of each of these two resulting masks represents VOls covering each particular kidney.

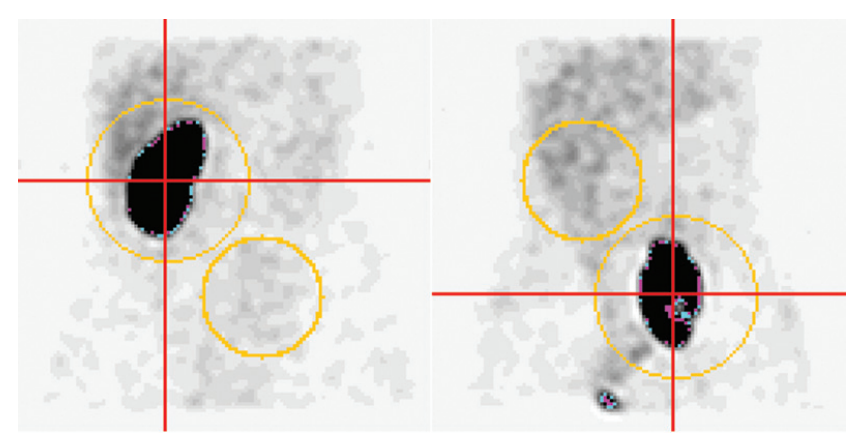

Figure 3. Split renal function assessment from SPECT study of the same patient as in Figure 2 demonstrated on the coronal view. Left and right image represents other coronal slices due to different antero-posterior location of kidneys. Each VOI was determined by an intersection of a corresponding sphere (orange circle on the slice) and an intensity mask (threshold denoted by dots on kidney's surface). The VOls are represented by the dark areas covering each particular kidney

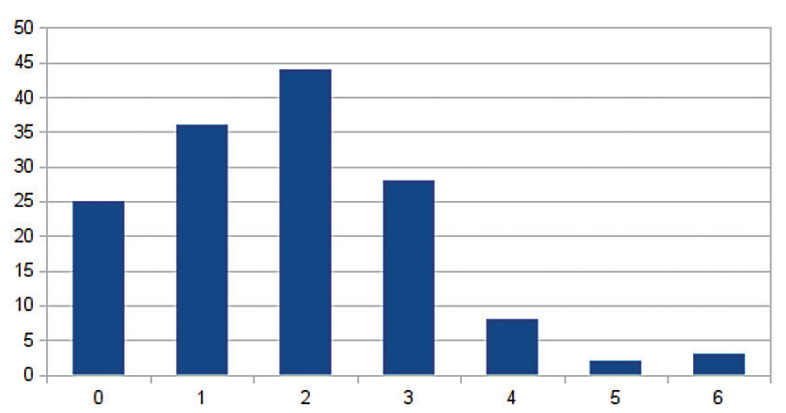

Figure 4. Distribution of absolute differences in terms of percentage points between SPECT and planar imaging within the patient study. Horizontal axis represents percentage points difference, vertical axis represents absolute numbers of patients with particular difference level (total 146)

No background correction similar to planar scintigraphy evaluation was applied. Total counts from each corresponding VOI came as inputs to the following formulas for split renal function:

(Equation 3) $R=\frac{N_{R}}{N_{R}+N_{L}} \cdot 100 \%$

(Equation 4) $L=\frac{N_{L}}{N_{R}+N_{L}} \cdot 100 \%$

where $N_{R}$ and $N_{L}$ were the total counts within the right and the left kidney $\mathrm{VOI}$, respectively.

\section{Evaluation}

Split renal function ratio within the interval 50/50\%-55/45\% was considered as normal, more than $55 / 45 \%$ as pathological finding. Normal kidneys had smooth outlines, no focal loss of the renal parenchyma or contractions and normal localization on both planar and SPECT images.

Finally, the values of the split renal function provided by all three methods in case of phantom study (methods A, B1, and B2) were compared to the real ratios of activities in the bottles assuming they had been accurately measured. 
Table 2. Results of the phantom study demonstrate comparison between uptake ratios provided by three imaging methods (planar, SPECT and SPECT CTAC) and measured relative activity. Values of the uptake ratio were calculated according equations 1 and 2 for planar imaging and according equations 3 and 4 for SPECT imaging. Different values are provided for both normal (N) and dystopic (D) geometry. Average and maximal absolute differences were calculated between particular method and measured relative activity and are represented in the terms of percentage points

\begin{tabular}{|c|c|c|c|c|c|c|c|c|}
\hline \multicolumn{2}{|c|}{ Relative activity (\%) } & \multirow[t]{3}{*}{ Geometry } & \multicolumn{6}{|c|}{ Uptake ratio (\%) } \\
\hline \multirow[b]{2}{*}{ Right } & \multirow[b]{2}{*}{ Left } & & \multicolumn{2}{|c|}{ Planar (A) } & \multicolumn{2}{|c|}{ SPECT (B1) } & \multicolumn{2}{|c|}{ SPECT CTAC (B2) } \\
\hline & & & Right & Left & Right & Left & Right & Left \\
\hline 50 & 50 & N & 51 & 49 & 51 & 49 & 51 & 49 \\
\hline 49 & 51 & D & 51 & 49 & 53 & 47 & 51 & 49 \\
\hline 60 & 40 & $\mathrm{~N}$ & 60 & 40 & 62 & 38 & 61 & 39 \\
\hline 60 & 40 & D & 61 & 39 & 59 & 41 & 60 & 40 \\
\hline 69 & 31 & $\mathrm{~N}$ & 69 & 31 & 71 & 29 & 71 & 29 \\
\hline 69 & 31 & D & 71 & 29 & 74 & 26 & 72 & 28 \\
\hline 80 & 20 & $N$ & 80 & 20 & 80 & 20 & 81 & 19 \\
\hline 80 & 20 & D & 80 & 20 & 83 & 17 & 81 & 19 \\
\hline 90 & 10 & N & 89 & 11 & 91 & 9 & 91 & 9 \\
\hline 90 & 10 & D & 88 & 12 & 92 & 8 & 91 & 9 \\
\hline \multicolumn{3}{|c|}{ Average absolute difference } & \multicolumn{2}{|c|}{0.9} & \multicolumn{2}{|c|}{2.1} & \multicolumn{2}{|c|}{1.3} \\
\hline \multicolumn{3}{|c|}{ Maximal absolute difference } & \multicolumn{2}{|c|}{2} & \multicolumn{2}{|c|}{5} & \multicolumn{2}{|c|}{3} \\
\hline
\end{tabular}

In case of the patient study the values of both methods (A and B1) were mutually compared.

The key parameter used to make any conclusion was the average absolute difference in terms of percentage points between corresponding sets of result values.

\section{Results}

\section{Phantom measurements}

Results of the comparison between evaluated split renal function and known activity ratios in the phantom bottles are presented in Table 2. It shows that there were no differences between particular imaging method and real data which would become clinically significant in case of real patient. The average value difference in terms of percentage points (pp) acted on behalf of planar imaging (0.9 pp), whereas SPECT method was noticeably worse (2.1 pp). Results of SPECT got considerably better (1.3 pp) when using attenuation correction. Also the maximal difference was lowest in the case of planar imaging (2 pp), whereas by SPECT it raised up to $5 \mathrm{pp}$, when using attenuation correction only to $3 \mathrm{pp}$.

\section{Patient study}

From the test group 83 patients had symmetrical (50/50\%$-55 / 45 \%)$ finding. The rest 57 patients had kidneys with their relative uptake ratio more than $5 \mathrm{pp}$ different from the ideal $50 / 50 \%$ level. Those findings (> 55/45\%) represent a pathological state.

In case of patients there was no possibility to get the real activity uptakes in the kidneys and the real uptake ratios resulting from that. Therefore it was only possible to compare the methods mutually.

In $17 \%$ of patients $(23 / 140)$ both methods provided identical separate renal function ratio. In $57 \%$ of patients (80/140) the methods diverged in range of $1-2 \mathrm{pp}$. For the rest $26 \%$ of patients $(37 / 140)$ the difference between results was greater or equal to $3 \mathrm{pp}$.
Table 3. Comparison of renal function ratios between method $A$ and $B$ in patients with normal (symmetrical) finding (ratios within the range of 50/50\%-55/45\%)

\begin{tabular}{|c|c|c|}
\hline \multirow[t]{2}{*}{$\begin{array}{l}\text { Difference between } \\
\text { methods }\end{array}$} & \multicolumn{2}{|c|}{$\begin{array}{c}\text { Numbers of patients with normal split } \\
\text { renal function }(<55 / 45 \%)\end{array}$} \\
\hline & Absolute & Relative to total (\%) \\
\hline No difference & 13 & 16 \\
\hline Difference in range of $1-2 \mathrm{pp}$. & 53 & 64 \\
\hline Difference in range of 3-6 pp. & 17 & 20 \\
\hline Total & 83 & 100 \\
\hline
\end{tabular}

Table 4. Comparison of renal function ratios between method $A$ and $B$ in patients with pathological finding (ratios greater than $55 / 45 \%$ )

\begin{tabular}{lcc} 
Difference between methods & \multicolumn{2}{c}{$\begin{array}{c}\text { Numbers of patients with pathological } \\
\text { split renal function }(>\text { 55/45\%) }\end{array}$} \\
\cline { 2 - 3 } & Absolute & Relative to total \\
\hline No difference & 10 & 18 \\
Difference in range of $1-2$ pp. & 27 & 47 \\
Difference in range of 3-6 pp. & 20 & 35 \\
Total & 57 & 100
\end{tabular}

According to Table 3 and Table 4, the most frequent difference between results of both methods was in the range of 1-2 pp regardless the kidney's pathology.

The average absolute difference between SPECT (method B1) and planar imaging (method $\mathrm{A}$ ) was $1.8 \mathrm{pp}$. The maximum absolute difference was 6 pp.

\section{Discussion}

The relative renal uptake of ${ }^{99 m} \mathrm{Tc}$-DMSA is valuable parameter and is used as an index of split renal function and to evaluate the 
renal function change at patient's follow up. The most accurate assessment of split renal function has always been at the forefront in many papers [14-17], in the interest of both nuclear medicine physicians and nephrologists and urologists.

99mTc-DMSA as a very good cortical imaging agent is preferentially taken up by the proximal renal tubule and first part of Henle's loop. According to recent papers $[12,18]$ the renal uptake of this tracer is dependent on the normal proximal tubule receptor - mediated endocytosis. Megalin and cubilin are cooperating receptors essential to the proximal tubule endocytic uptake of proteins from the glomerular filtrate in the proximal tubule.

${ }^{99 m}$ Tc-DMSA renal scan is still considered as the gold standard for diagnosing acute pyelonephritis and chronic lesions like renal scars and is recommended in many investigative algorithms in pediatric practice $[10,11,19-21]$.

For a more detailed renal parenchyma imaging basal investigation (anterior, posterior, left oblique posterior and right oblique posterior projections) is complemented using pinhole collimator images or SPECT. We prefer pinhole images in young children up to 5 years and SPECT examination for older children and adults.

The quantification of the split renal function can be performed by different mathematical analysis, most often from planar images with compensation for kidney depth based on the geometric mean, which can be determined using anterior and posterior views [15]. In context of quantitative ${ }^{99 m}$ Tc-DMSA imaging, it has been reported that the kidney depth compensation is unnecessary when calculating differential renal function for normally located kidneys in the pediatric population [16]. The geometric mean correction is less important in children than in adults, since children have a smaller renal depth. Hervas et al [15] conclude that for children less than 10 years it is unnecessary to perform depth correction using the geometric mean except in cases of major malformations and position anomalies.

In this study the group of patients included 57 children and adolescents with asymmetrical kidneys due to a reduced renal function or different size or abnormal renal position. Therefore the compensation for kidney depth was not avoided.

The fact that in our phantom study the planar imaging provided more accurate split renal function results than tomography (especially plain SPECT) is in contradiction to our primary assumption of SPECT superiority over the planar imaging. According to the comparison between SPECT and SPECT CTAC, the probable reason may be just the effect of attenuation. The attenuation correction, which acts similarly like the geometric mean for planar imaging, is used to correct the underestimated uptake due to particular kidney depth. Therefore the attenuation correction should make the results more close to real values which was supported by our phantom study. Unfortunately, CT is strongly inadvisable for children and adolescents due to an indispensable radiation burden. However, even with CTAC the planar scintigraphy still seems to be slightly better. The possible reason arises from dependency of both methods on $\mathrm{ROI} / \mathrm{NOI}$ determination.

In case of SPECT and its parameter-based VOI, the procedure is quite straightforward and independent of kidney size, shape and position. There is sufficient degree of freedom in defining the spherical masks with no influence to the final VOIs. The only parameter which directly influences the results and therefore is important for reproducibility is the intensity threshold. It is subject to an individual decision, similarly like free-hand ROI drawing within the planar image assessment. It seems that delineating the VOI based on the intensity threshold is less accurate than drawing manual $\mathrm{ROI}$ on the planar image. However, defining the VOI slice by slice like by radiotherapy planning would probably bring no benefit but more uncertainties instead.

Based on the facts provided by the phantom study and also because the percentage points differences within the patient study were mostly under the threshold of any clinical impact (less than or equal to $5 \mathrm{pp}$ ) [15], it is possible to keep practice of planar imaging without any risk of mistake. Also the simplicity and shorter acquisition speak for it.

In the light of these facts ${ }^{99 m} \mathrm{Tc}-\mathrm{DMSA}$ planar imaging still seems to play an important role in the chain of split renal function assessment.

\section{Conclusion}

According to our experience in the evaluation of the split renal function using planar and tomographic imaging for both phantom and patient study, it can be concluded that split renal function assessment performed by planar examination with geometric mean as the kidney depth compensation is valid for common routine pediatric practice including cases with atypical renal localization. The results of planar imaging are at least equivalent or even better than results of tomographic studies. There is no need to update the established procedure.

\section{References}

1. Piepsz A, Blaufox MD, Gordon I et al. Consensus on renal cortical scintigraphy in children with urinary tract infection. Semi Nucl Med 1999; 29: 160-174.

2. Lin KY, Chiu NT, Chen MJ et al. Acute pyelonephritis and sequelae of renal scar in pediatric first febrile urinary tract infection. Pediatr Nephrol 2003; 18: 362-365

3. Simsek E, Kologlu N, Yugunt I et al. Comparison of imaging studies and diagnostic tests for the diagnosis and follow-up of recurrent urinary tract infection in children. Int Pediatr 2004; 19: 109-113.

4. Camacho V, Estorch M, Fraga G. DMSA study performed during febrile urinary tract infection: a predictor of patient outcome? Eur J Nucl Med Mol Imaging 2004; 31: 862-866.

5. Moorthy I, Easty M, McHugh K et al. The presence of vesicoureteric reflux does not identify a population at risk for renal scarring following a first urinary tract infection. Arch Dis Child 2005; 90: 733-736.

6. Zaki M, Badawi M, Al Murati G et al. Acute pyelonephritis and renal scarring in Kuwaiti children: a follow-up study using 99mTc DMSA renal scintigraphy. Pediatr Nephrol 2005; 20: 1116-1119

7. Gonzales E, Papazyan JP, Giardin E. Impact of vesicoureteral reflux on the size of renal lesions after an episode of acute pyelonephritis. J Urol 2005; 173: $571-474$.

8. Hoberman A, Charron M, Hickey RW et al. Imaging studies after a first febrile urinary tract infection in young children. N Engl J Med 2003; 348: 195-202.

9. Piepsz A. Cortical scintigraphy and urinary tract infection in children. Nephrol Dial Transplant 2002; 17: 560-562.

10. Chroustova D, Palyzova D, Urbanova I, Kolska M. Results of a five-year study of 99mTc-DMSA renal scintigraphy in children and adolescents following acute pyelonephritis. Nuclear Medicine Review 2006; 9: 46-50.

11. Piepsz A, Colarinha P, Gordon I et al. Gudelines on 99mTc-DMSA scintigraphy in children. Eur J Nucl Med 2001; 28: 37-41.

12. Taylor T. A. Radionuclides in Nephrourology, Part1: Radiopharmaceuticals, Quality Control, and Quantitative Indices. J Nucl Med 2014; 55: 608-615. 
13. Holm S, Borgwardt L, Loft A et al. Paediatric doses - a critical appraisal of the EANM paediatric dosage card. Eur J Nucl Med Mol Imaging 2007; 34: 1713-1718.

14. Tsukamoto $\mathrm{E}$, Itoh $\mathrm{K}$, Katoh Ch et al. Validity of 99mTc-DMSA renal uptake by planar posterior-view method in children. Annals of Nuclear Medicine 1999; 13: 383-387.

15. Hervás I, Martí JF, Gonzáles A, Ruiz JC et al. Is the depth correlation using the geometric mean really necessary in a99mTc-DMSAscan in the paediatric population? Nuclear Medicine Communication 2001; 22: 547-552.

16. Lythgoe MF, Gradwell MJ, Evans K, Gordon I. Estimation and relevance of depth correction in paediatric renal studies. Eur J Nucl Med 1998; 25: 115-119.

17. Weinberger $S$, Bäder $M$, Scheurig-Münkler $C$ et al. Optimizing evaluation of split renal function in a living kidney donor using scintigraphy and calculation of the geometric mean: A case report. Case Rep Nephrol Urol 2014; 4: 1-4.

18. Weyer K, Nielse R, Petersen SV et al. Renal uptake of 99mTc-Dimercaptosiccinic acid is dependent on normal proximal tubule receptor — mediated endocytosis. J Nucl Med 2013; 54: 159-165.

19. Hossain MM Urinary tract infection in children: importance of proper diagnosis and management. Bangladesh J Child Health 2013; 37: 1-4.

20. Prasad MM, Cheng EY. Radiographic evaluation of children with febrile urinary tract infection: Bottom-up, top-down, or none of the above? Hindawi Publishing Corporation Advances in Urology Article 2012; ID 716739, 8 pages doi: 10.1155/2012/716739.

21. Biassoni L, Chippington S. Imaging in urinary tract infection: Current strategies and new trends. Semin Nuc Med 2008; 38: 56-66 